\title{
Los Estados en los márgenes: soberanía y gubernamentalidad en el principal valle cocalero peruano
}

\section{Sofia Isabel Vizcarra Castillo* Christoph Heuser** (i)}

\section{Resumen}

La gobernanza de zonas con presencia de economías ilegales es parte de una amplia discusión académica, sin embargo es poco lo que se discute específicamente sobre las características del Estado en estas zonas. Por ello, a partir de un estudio de caso cualitativo, este artículo examina la interacción entre la intervención estatal en el Valle de los ríos Apurímac, Ene y Mantaro (VRAEM) y su objeto de gobierno. Esta zona es la principal cuenca cocalera del Perú y con presencia de remanentes terroristas, por lo que constituye una de las principales preocupaciones de seguridad del gobierno peruano. Gobernar el VRAEM ha sido siempre un desafío y no siempre se ha realizado según los planes del propio gobierno. Basado en conceptos de Foucault, este trabajo muestra que los habitantes del VRAEM son gobernados por al menos dos tipos de poderes desde el Estado: un poder soberano y un poder gubernamental. La convivencia entre estos tipos de poderes permite explorar las multiplicidades de prácticas gubernamentales existentes en la zona, así como las resistencias locales a ambos tipos de poder.

Palavras clave: Perú, Estado, gubernamentalidad, poder soberano, narcotráfico.

* Pontificia Universidad Católica del Perú, Lima, Perú.

** German Institute of Global and Area Studies - GIGA, Alemanha. 


\section{The states in the fringes: sovereignty and governmentality in the main Peruvian coca growing region}

\section{Abstract}

Governance of areas with prevalence of illegal economies belongs to a broad academic discussion, even though studies on the characteristics of the State in those areas are scarce. Based on a qualitative case study, this paper examines the interactive consequences of state intervention in the Valley of the Apurímac, Ene and Mantaro Rivers (VRAEM). This region is the main coca growing area of Peru and operational center for terrorist remnants. For this reason, the region constitutes one of the main security concerns of the Peruvian government. Governing the VRAEM has always been a challenge and has not always been carried out according to government's plans. This paper shows that due to the rationale of intervening in an area of illicit economies the VRAEM is governed by at least two types of State power: a sovereign power and a governmental power. The coexistence of these types of state powers allows exploring the multiplicities of governmental practices in the area, as well as the local resistance to both powers.

Keywords: Peru, state, governmentality, sovereign power, drug-trafficking.

\section{Introducción ${ }^{1}$}

n varios de los Estados del mundo existe lo que Guillermo O’Donnell
(1993) denominó "zonas marrones", territorios dentro del Estado
pero solo parcialmente bajo control estatal. Recientes investigaciones vinculan estas regiones donde el Estado solo tiene una influencia fragmentada con zonas peligrosas y "desordenadas" (Felbab-Brown; Trinkunas; Hamid, 2017). En estas zonas, se ha estudiado la aparición de ordenes híbridos en los que el poder se ejerce de manera compartida entre actores estatales y no-estatales, en varios casos, ilegales (Arjona, 2017; Boege; Brown; Clements, 2009). Si bien estos estudios contribuyen a explicar las diferentes formas de gobernanza local, no son suficientes para comprender al Estado,

\footnotetext{
${ }^{1}$ Los autores agradecen los comentarios de los revisores que han servido para mejorar el presente artículo así como para plantear futuras reflexiones e investigaciones. Las omisiones en este artículo son enteramente responsabilidad de los autores.
} 
como actor principal pero diverso, y los efectos de sus diferentes formas de intervención en este tipo de áreas. Este artículo tiene por objetivo ilustrar el análisis de la intervención estatal en estas "zonas marrones" usando el concepto de gubernamentalidad de Foucault aplicado al caso del Valle de los Ríos Apurímac, Ene y Mantaro (VRAEM).

EI VRAEM es una de esas regiones que la opinión pública, la prensa (Paredes Castro, 2017) e incluso algunos académicos (Calmet; Salazar, 2013; Carpio; Annoni, 2016) consideran "al margen del Estado" (Das; Poole, 2004). Se ubica en el centro sur del Perú, su territorio comprende 69 distritos, en 10 provincias de 05 regiones del país. Como varias de las denominadas zonas marrones, el VRAEM está marcado por la existencia de una fuerte economía ilegal ligada a la producción de derivados cocaínicos. Para el año 2016, el VRAEM era el mayor valle cocalero del Perú con un total de 20304 hectáreas de coca cultivadas, representando 70\% de la producción potencial nacional (UNODC, 2017). Por otra parte, el VRAEM también había sido el refugio de los remanentes del movimiento terrorista Sendero Luminoso², luego de la captura de su líder en 1992. Estos componentes lo han convertido en un territorio prioritario para la intervención estatal por motivos de seguridad. Sin embargo, esta zona no corresponde a una división administrativa del país, sino a un recorte territorial paulatinamente definido por los estados de emergencia y los planes de intervención planteados por el gobierno, a partir del año 2003, frente a la doble amenaza del narcotráfico y el terrorismo.

La presencia de estas actividades podría haber convertido al VRAEM en una zona liberada al margen del Estado definida por actores non-estatales y por la economía ilícita. Estas zonas están caracterizadas frecuentemente por problemas de funcionamiento del orden democrático, por la acción de actores paraestatales o una inexistencia de la presencia estatal. Diversos autores han explorado recientemente el surgimiento de este tipo de zonas en

${ }^{2} \mathrm{~A}$ lo largo del artículo haremos uso del término terrorista para referirnos a los remanentes de esta organización subversiva. Tanto la legislación peruana como las autoridades oficiales usan dicha denominación para referirse a los integrantes de este grupo. 
América Latina, como las favelas de Río de Janeiro (Arias, 2017), las comunas de Medellín (Duncan, 2013) o varias regiones en México (Lessing, 2017; Pansters, 2018). Sin embargo, esto no es lo que ha sucedido en el VRAEM. A pesar de que algunos autores asocian la presencia de la economía ilegal a la debilidad e incluso ausencia del Estado en la zona (Carpio; Annoni, 2016), nosotros proponemos matizar estas afirmaciones. Nuestro objetivo es interrogarnos sobre esta supuesta debilidad y analizar en mayor detalle la presencia del Estado en el VRAEM.

Lo que existe actualmente en el valle es una zona que ha conocido un sostenido crecimiento económico y urbano, con niveles de violencia controlados y tasas de homicidio bajas - especialmente si se comparan con otras zonas en las que está presente el narcotráfico. Allí, el Estado ha aumentado su presencia sin que ello haya representado una disminución de la presencia de actividades criminales en la zona (Dargent, 2012).

A pesar de que, en las últimas décadas, la imposición de un orden por la fuerza haya sido dificultosa, el surgimiento de indicios de otras formas de acción gubernamental desde las burocracias civiles muestra que el Estado está presente en la vida de los individuos que allí habitan. La pregunta que surge es, entonces, ¿En qué medida el Estado está efectivamente presente en el VRAEM? Y, en general, ¿Cómo se ha gobernado el VRAEM en las últimas décadas? ¿Cuáles son las prácticas estatales que allí se evidencian y como las perciben sus habitantes?

Planteamos que, desde la perspectiva estatal, han existido al menos dos maneras de gobernar el VRAEM que conviven hasta la actualidad. La primera se ejerce controlando el territorio a través del Estado de emergencia. La segunda, desde la población, intentando generar "bienestar", convirtiendo en beneficiarios de políticas socioeconómicas a quienes allí residen. Exploraremos, a partir de los conceptos propuestos por Foucault sobre soberanía y gubernamentalidad, como conviven estos dos tipos de prácticas estatales en el VRAEM y como los ciudadanos que allí residen las experimentan. Desde una perspectiva más general, cuestionamos la 
correlación que se realiza entre fragilidad del Estado y el desarrollo de economías ilegales a partir de un análisis plural del Estado.

A fin de responder a estas interrogantes, empleamos métodos cualitativos que incluyeron i) revisión documental tanto de reportes gubernamentales como normas estatales; ii) entrevistas semiestructuradas a agentes públicos (policías nacionales, policía municipal, militares y ex militares, servidores públicos civiles) y habitantes locales (comerciantes locales, agricultores, contrabandistas, entre otros); iii) etnografía de las relaciones entre agentes estatales y ciudadanos en cuatro centros urbanos de la región. El trabajo de campo se realizó en diferentes etapas entre los años 2015 y $2017^{3}$.

El artículo presenta, en primer lugar, una discusión del Estado desde la perspectiva foucaultiana y plantea una base teórica para su análisis. En segundo lugar, analizamos, desde la perspectiva del poder soberano, las diferentes estrategias que el Estado ha implementado en la zona y su vínculo con el estado de emergencia. Finalmente, describimos la presencia del Estado desde una perspectiva gubernamental y sus efectos en la población local.

\section{Aproximaciones al Estado desde Foucault}

Foucault enfoca el tema del Estado desde el estudio de las formas de gobierno de si y de los otros mostrando los cambios en los objetos y los saberes del ejercicio del poder. Poder y saber son indivisibles en la lógica de Foucault, y en la manera en la que se realiza el asujetamiento de los individuos.

Este asujetamiento significa la manera en la que el individuo se convierte en sujeto de gobierno, situándolo al centro de las relaciones de poder, y la manera en la que él acepta ser gobernado. Las prácticas estatales han cambiado en función del objeto y de quien ejerce esas prácticas. Como lo

${ }^{3}$ Realizamos investigaciones del campo durante el mismo periodo en las municipalidades de Pichari, Kimbiri, Llochegua y Santa Rosa, con entrevistas complementarias en Lima y Ayacucho. El desarrollo de la investigación siguió los lineamientos de los comités de ética de las respectivas instituciones. En ese sentido, se procedió a atribuir nombres ficticios y mantener en el anonimato la identidad de los entrevistados para su protección. 
señala Garmany (2009, p. 726), siguiendo el argumento de Timothy Mitchell, "el Estado foucaultiano es un 'efecto' [...] constituido por el indivisible de las mismas prácticas (incluyendo el lenguaje y el discurso) que constituyen otras estructuras aparentes como la economía y la sociedad".

Lo que propone Foucault es ir más allá de la hegemonía del modelo jurídico-político del Estado ligado a la soberanía, y entenderlo como una serie de posibilidades ligadas a sus prácticas, definidas como las acciones que realiza en dirección al objeto del poder (García Romanutti, 2014). Si bien su objeto de estudio no fue el Estado, se entiende desde su perspectiva que este es un actor que ejerce prácticas de poder y que busca imponerse sobre otros actores.

Foucault traza los diferentes objetos que han sido el centro de las prácticas de poder desde una perspectiva genealógica, identificando diferentes tipos de poder - el poder soberano, el poder disciplinar y el poder gubernamental - que corresponden a prácticas y objetos de poder diferenciados. Esta perspectiva nos permite hablar no de un Estado, sino de los Estados o, más precisamente, de las prácticas de estatalidad ligadas a diferentes formas de ejercer el poder. Reconocemos que el poder disciplinar está fuertemente presente en el VRAEM - principalmente para combatir el narcotráfico y los remanentes de Sendero Luminoso. Pero nos centramos, principalmente, en el poder soberano y en el gubernamental, ya que, desde una perspectiva histórica linear, parecen corresponder a paradigmas diferentes de Estado. Desde una observación contextualizada, se puede evidenciar una convivencia de varios tipos de poderes. Es necesario recordar que Foucault (2006) entiende poder como una relación entre sujeto y objeto, como se puede también entender la relación entre la sociedad y el Estado. En otras palabras, la relación entre Estado y población no es únicamente una cuestión de control, sino de comunicación mutua y de reconocimiento del Estado como un actor legítimo.

La identificación del poder soberano se realiza en la época medieval, su principal objetivo era controlar el territorio a través de la fuerza. Es un poder que se impone y no tiene por objetivo el control de los individuos, a no ser 
que ello altere el control del territorio. En cambio, el poder gubernamental surge en la época moderna, con el objetivo de mejorar la población (Foucault, 2006). El cambio no solo implica cambiar el objetivo sino la forma en la que se ejerce el poder. A diferencia del poder soberano que se impone a los individuos, el poder gubernamental es ejercido por los propios individuos para regularse a sí mismos (Foucault, 2000).

En ese sentido, el poder gubernamental es sobre todo una forma de autorregulación individual que lleva a que la conducta del individuo se oriente hacia su mejoría, o a lo que las normas y regulaciones hayan definido como mejoras deseables. El rol del sujeto y su libertad son centrales en el pensamiento de Foucault, no es tanto sobre el poder, sino sobre el asujetamiento voluntario (Adorno, 2017). Pensar, entonces, la gubernamentalidad implica poder pensar la presencia del Estado, incluso cuando este no esté físicamente (a través de infraestructura, burocracias etc.) presente en un lugar (Garmany, 2009). Esta mirada al Estado y a la gubernamentalidad nos permitirá explorar, en el caso del VRAEM, la presencia de dos poderes que parecen convivir desplegando diferentes dispositivos.

\section{El Estado de emergencia: mecanismo del poder soberano}

\section{Evolución de los enfoques de intervención del Estado en el VRAEM}

El VRAEM fue uno de los teatros del conflicto armado interno que vivió Perú durante la década de los ochenta. Durante ese periodo, se instaló una economía de guerra basada en el narcotráfico. La cocaína era el único producto que tenía una salida asegurada al mercado incluso durante las épocas más álgidas del conflicto. Tras la derrota de Sendero y con el retorno a la democracia, en el año 2001, se intenta que el país vuelva a la normalidad. Sin embargo en mayo del 2003, el presidente Alejandro Toledo declaró en emergencia todo el territorio nacional, luego de una serie 
de huelgas en el país. Luego de 90 días, la declaratoria de emergencia se mantiene solo en algunos distritos del país. Estos distritos corresponden a lo que ahora denominamos VRAEM, que continúan declarados en emergencia.

El estado de emergencia es una figura legal que se refiere a "la suspensión de la ley por el Estado soberano en nombre de la defensa o seguridad nacional" (Hanafi; Long, 2010, p. 147). Es decir, se suspende el orden constitucional con la finalidad de salvarlo del colapso y asegurar su soberanía ${ }^{4}$. Sin embargo, Agamben (2005) añade que este mecanismo, si se implementa de manera permanente, conlleva como peligro disminuir el orden legal democrático. El estado de emergencia es un mecanismo que ha sido frecuentemente usado en la historia republicana del Perú que Siles Vallejos califica como "la persistencia histórica de la excepcionalidad" con la siguientes características:

(i) conceden al Poder Ejecutivo potestades excepcionales de actuación y regulación; (ii) alteran el cuadro usual de división de poderes, concentrándolos en el Gobierno; (iii) habilitan la suspensión del ejercicio de determinados derechos fundamentales; $y$, (iv) permiten recurrir a las Fuerzas Armadas, en sustitución de la Policía, para vencer el peligro que se cierne sobre el país (Siles Vallejos, 2015, p. 74).

En el VRAEM, se despliegan tres de estas cuatro características, ya que no existe una concentración de poderes. Asimismo se suspenden las garantías y ciertos derechos constitucionales y se otorga el control del orden interno a las fuerzas de seguridad. En los últimos catorce años, lo único que ha variado en esta situación es la cantidad de distritos en emergencia y el detentor del orden interno (variando entre Policía Nacional y Fuerzas Armadas), sin cambiar la racionalidad de la excepción como se aprecia en el gráfico 1.

\footnotetext{
${ }^{4}$ Agamben reacciona a Carl Schmitt, quién definió al actor que determina el estado de emergencia como "el soberano" (Schmitt, 1996, p. 11).
} 
Gráfico 1: Declaratorias de Estados de Emergencia (por fecha y número de distritos declarados en emergencia y responsables del orden interno) y creación de nuevos distritos en el VRAEM

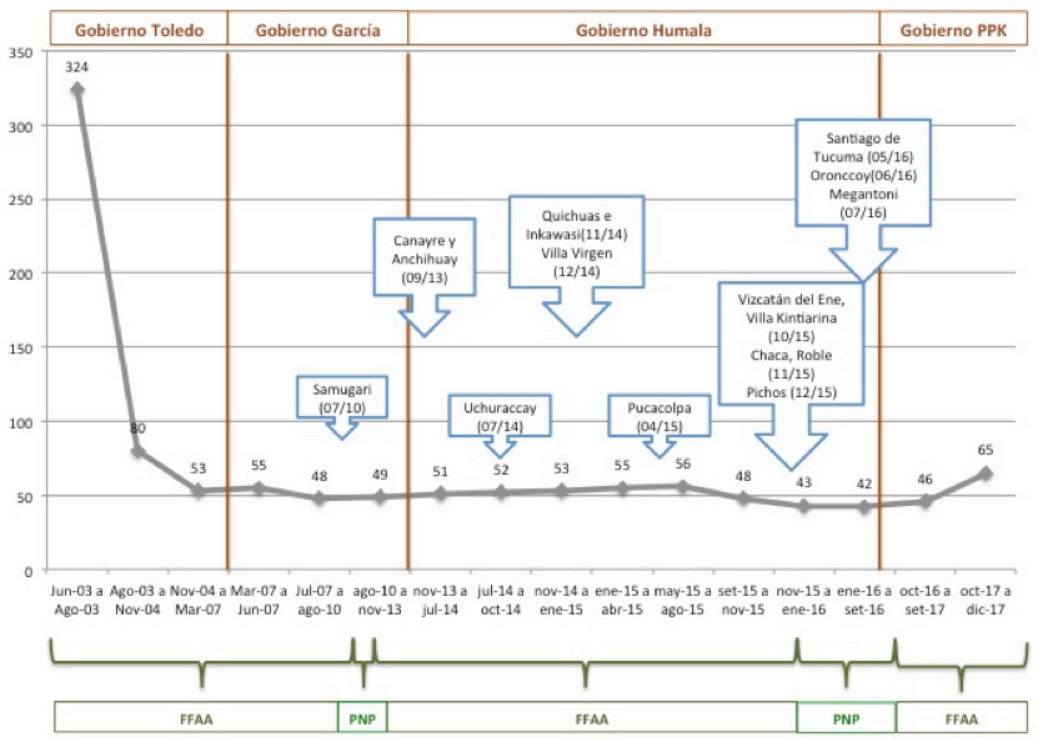

Leyenda: El gráfico muestra la cantidad de distritos declarados en estado de emergencia en el VRAEM (eje vertical) en función de las fechas de validez de los decretos de emergencia emitidos por el Estado peruano durante los diferentes gobiernos entre los años 2003 y 2017 (eje horizontal). Asimismo se resalta la creación de nuevos distritos en los departamentos que componen el VRAEM (señalizados con flechas) y también se evidencia los cambios del control del orden interno (debajo del eje horizontal), mostrando la alternancia en el control del orden interno del VRAEM entre las Fuerzas Armadas (FFAA) y la Policía Nacional del Perú (PNP).

Fuente: Elaboración propia en base a decretos supremos que declaran Estados de Emergencia disponibles en el SPIJ 
Las autoridades centrales tienen la idea de contener el problema de seguridad a un territorio determinado: el VRAEM, evitando así que la amenaza se expanda ${ }^{5}$. Es una política de contención territorial apoyada por diversos sectores en el país. En Lima, la capital de la República, a miles de kilómetros de distancia así como en el resto de las ciudades del país, el 95\% de los encuestados (encuesta urbana) consideraba que la presencia de movimientos terroristas en el VRAEM representaba un peligro y la mayoría consideraba que se financiaban a través del narcotráfico por lo que reclamaban "mano dura" contra el problema (IPSOS, 2012). Esta concentración en los temas de seguridad generó un dilema para el Estado.

\section{El dilema entre perseguir y proteger}

El poder soberano tiene por principal objeto el control de un territorio, sin necesariamente preocuparse por el bienestar de la población que allí radica. Durante la primera década de los años 2000, la presencia del Estado en la zona estuvo principalmente representada por las instituciones militares y policiales. Varios de los críticos del gobierno y la prensa apuntaban a la debilidad del Estado como la principal causa de los problemas de seguridad en el VRAEM. En ese sentido, apuesta por fortalecer su presencia, en primer lugar armada. Así, entre los años 2007 y 2010 se crean la región militar y el Frente Policial VRAE así como una escuela militar y policial en la zona. Se incrementó progresivamente el número de bases militares, reactivando algunas que habían sido usadas durante el conflicto armado interno; incluso existía el proyecto de crear un aeródromo militar en el valle (Monteagudo, 2013). También se incrementó el número de efectivos en servicio en la zona. El VRAEM se convirtió en una región "atractiva"

\footnotetext{
${ }^{5}$ En ese sentido, en el Estado de emergencia, los habitantes del territorio declarado en emergencia no son concebidos como ciudadanos. $\mathrm{O}$ al menos no como ciudadanos cuyo bienestar hay que priorizar, Desde la perspectiva del Estado, estos ciudadanos están al margen de los modos de producción y consumo (legales) y se les entiende como parte del problema. El Estado no los abarca, pero, por medio del ethos colectivo, legitima la violencia y consagra la inexistencia de políticas que prioricen el bienestar de la mayoría en dicha región.
} 
dónde prestar servicios policiales o militares ya que estar destacado allí representa un bono económico así como puntos adicionales. También se realizaron algunas mejoras en la infraestructura y el equipamiento de las fuerzas armadas.

La presencia del narcotráfico y del terrorismo también justificó el aumento de una burocracia estatal orientada a la implementación de políticas de control de drogas. Así, a la presencia militar se suma la presencia policial, particularmente de divisiones especializadas como la policía antidrogas o las divisiones de inteligencia y lavado de activos, así como Fiscalías y Juzgados Especializados y programas Desarrollo Alternativo. Asimismo, se otorga facultades a las fuerzas armadas para que intervengan en la lucha contra el narcotráfico.

Tanto terroristas como narcotraficantes son el objetivo principal de las acciones desplegadas por el Estado. La principal tarea es la interdicción y la persecución de estos delitos graves, incluso si ello no coincide necesariamente con las prioridades de la población local, cuyas preocupaciones están relacionadas con la delincuencia común. Este enfoque afecta la percepción local sobre los actores estatales.

Así, por ejemplo, un transportista de Pichari narraba la historia de un vecino cuya hija de 11 años había sido violada por un mototaxista ${ }^{6}$ de la zona. Cuando la policía capturó al violador, este último denunció al padre de la víctima como dueño de pozas de maceración de drogas. Enseguida, la policía desplegó un operativo para ir a destruir las pozas y capturar a los "narcotraficantes". La víctima y su familia tuvieron que huir y el violador fue liberado por la información que proporcionó. En este escenario, el poder soberano cumplió su rol persecutor contra aquel que era definido como el principal enemigo. La selectividad penal se aplicó para enfatizar la persecución de los delitos ligados a drogas e ignorar aquellos relacionados a seguridad ciudadana. Ello lleva a varios ciudadanos locales

${ }^{6}$ Un mototaxista es un conductor de un mototaxi, vehículo de tres ruedas compuesto por una motocicleta y cabina de pasajeros, también conocido en otros países como "touk-touk". Es un modo de transporte muy popular en la Amazonia peruana. 
a pensar que las fuerzas del orden no los protegen, sino que constituyen un enemigo que perjudica sus intereses económicos y sociales. Un taxista local, entrevistado durante el trabajo de campo en Kimbiri, lo resume así: "la policía no toma interés en casos de asaltos, pero cuando es droga, ahí si actúan rápido." (Tomás, Ayacucho, enero de 2016). Asimismo, un comerciante de Llochegua: "Mira voy a ser sincero, aquí la gente odia a la policía porque no interviene cuando hay problemas de robo, pero si interviene en cuestiones de narcotráfico y la gente vive de eso." (Fernando, Llochegua, octubre 2016). Este malestar erosiona la legitimidad de las fuerzas del orden a nivel local.

Las fuerzas del orden persiguen a cualquiera que cumpla con el perfil del enemigo, en el caso del VRAEM, de un narcoterrorista ${ }^{7}$. ¿Quién es el narcoterrorista? Es producto del difuminado de las fronteras entre estos dos delitos y su asociación sistemática. En el VRAEM, esta construcción del enemigo se amplía a todo aquel vinculado al narcotráfico, por lo que toda la población termina siendo sospechosa. Al ya no haber distintivos para reconocer al narcoterrorista, se crea una discrecionalidad de la persecución a criterio de las fuerzas del orden.

El énfasis en la persecución a los delitos de drogas y terrorismo en detrimento de otro tipo de delitos también se explica porque la estrategia prioriza logros operativos en estos dos temas. Las inversiones más importantes luego del rubro infraestructura están destinadas al sector de defensa y lucha contra el terrorismo. Este rubro suma 30,4\% de las inversiones, mientras que el siguiente punto, inclusión social, solo representa el 6,6\% (Donadio, 2016). A nivel nacional, no se evalúa el rol de la policía y de las fuerzas

\footnotetext{
${ }^{7}$ El término narcoterrorismo es de uso común en la prensa peruana desde hace varios años, como se puede apreciar en diferentes notas periodísticas (por ejemplo: https://andina.pe/ agencia/noticia-jefe-del-comando-conjunto-las-ff-aa-pide-a-poblacion-confiar-estrategiacontra-narcoterrorismo-248069.aspx). Sin embargo, el Comando Conjunto de las fuerzas armadas, en sus notas institucionales, siempre hace referencia a "delincuentes terroristas". No obstante, en su entendimiento del problema del VRAEM, tanto narcotráfico como terrorismo están considerados con el mismo nivel de amenaza (Para mayor detalle leer "Por qué existe el VRAEM?" CCFFAA disponible en: http://www.ccffaa.mil.pe/menuDEFENSA/ vraem.html).
} 
armadas por los indicadores de seguridad ciudadana en el VRAEM. Por el contrario, se espera tener logros en la lucha contra el "narcoterrorismo", por lo que se visibilizan las capturas de terroristas, la destrucción de pozas, laboratorios y pistas clandestinas así como las grandes incautaciones de drogas. Asimismo, el presupuesto de ambos cuerpos se sustenta en dichos avances operativos, por lo que es de especial interés mejorar en estos indicadores. Así, se deja de lado la persecución de delitos como el tráfico de insumos químicos o el lavado de dinero que son más difíciles de probar y representan menores logros en términos operativos (Vizcarra, 2014).

\section{Hacia el surgimiento de una gubernamentalidad en el VRAEM}

\section{La población del VRAEM y el desarrollo de un Estado protector mínimo}

Durante la larga intervención estatal en el VRAEM, hemos observado cambios importantes en la estrategia. A partir del año 2006, el gobierno comienza a pensar al VRAEM como un territorio en el cual se debe intervenir de manera integral y no solo con fuerzas del orden. Es a partir de ese año que se formulan sucesivas estrategias de intervención - los denominados "planes VRAEM" que van ajustando sus contenidos, como se aprecia en el cuadro 1. Durante sus primeros años (2007 - 2009), la estrategia estuvo liderada por el Ministerio de Defensa aunque contemplaba un componente de desarrollo socioeconómico. En el año 2009 se opta por pasar el liderazgo a una instancia civil, con la creación de la Secretaría del Grupo de Trabajo Multisectorial del VRAEM en la Presidencia del Consejo de Ministros (PCM). A partir de este momento, se comienza a fortalecer la presencia de aparatos burocráticos civiles. Además, entre el 2009 y el 2012, el Plan Integral Territorial enfatiza el componente de comunicación y participación de la población local. Sin embargo, ello no logra contrarrestar el malestar por la militarización de la zona ni frenar la aparición de conflictos sociales 
relacionados a la oposición a la política de drogas. Durante el gobierno García, no se registran cambios sustanciales en los resultados de seguridad y socioeconómicos en el VRAEM.

Cuadro 1: Estrategias de intervención del gobierno central en el VRAEM.

\begin{tabular}{|c|c|c|c|}
\hline Vigencia & Nombre de la Iniciativa & Ejes & $\begin{array}{l}\text { Institución } \\
\text { Responsable }\end{array}$ \\
\hline $\begin{array}{l}2006 \\
\text { (Relanzado } \\
\text { en 2007) - } \\
2009\end{array}$ & $\begin{array}{l}\text { Esquema de } \\
\text { intervención estratégica } \\
\text { integral denominado } \\
\text { "Una opción de Paz y } \\
\text { Desarrollo en Seguridad } \\
\text { para el Valle de los Ríos } \\
\text { Apurímac y Ene - Plan } \\
\text { VRAE" }\end{array}$ & $\begin{array}{l}\text { Militar } \\
\text { Policial } \\
\text { Desarrollo } \\
\text { Socioeconómico }\end{array}$ & $\begin{array}{l}\text { Secretaría del } \\
\text { Grupo de Trabajo } \\
\text { Multisectorial del } \\
\text { VRAE (adscrita al } \\
\text { sector Defensa) }\end{array}$ \\
\hline $\begin{array}{l}2009- \\
2012\end{array}$ & $\begin{array}{l}\text { Plan Integral Territorial } \\
\text { VRAE: 2009-2012 }\end{array}$ & $\begin{array}{l}\text { Desarrollo económico } \\
\text { Seguridad y legalidad } \\
\text { Comunicación y } \\
\text { participación }\end{array}$ & $\begin{array}{l}\text { Secretaría del } \\
\text { Grupo de Trabajo } \\
\text { Multisectorial del } \\
\text { VRAE (adscrita a } \\
\text { la Presidencia del } \\
\text { Consejo de Ministros) }\end{array}$ \\
\hline $\begin{array}{l}2013- \\
2016\end{array}$ & $\begin{array}{l}\text { Programa de } \\
\text { Intervención } \\
\text { Multisectorial }\end{array}$ & $\begin{array}{l}\text { Lucha contra la } \\
\text { pobreza } \\
\text { Lucha contra la } \\
\text { desigualdad } \\
\text { Lucha contra el tráfico } \\
\text { ilícito de drogas y las } \\
\text { bandas criminales } \\
\text { organizadas } \\
\text { Lucha contra el } \\
\text { terrorismo }\end{array}$ & $\begin{array}{l}\text { Comisión } \\
\text { Multisectorial para } \\
\text { la Pacificación y el } \\
\text { Desarrollo Económico } \\
\text { social en el VRAEM } \\
\text { - CODEVRAEM } \\
\text { (adscrita a la } \\
\text { Presidencia del } \\
\text { Consejo de Ministros) }\end{array}$ \\
\hline
\end{tabular}

Fuente: Elaboración propia en base a documentos oficiales.

Durante el gobierno Humala, la intervención en el VRAEM, plasmada en el "Programa de Intervención Multisectorial 2013 - 2016" (CEPLAN, 2012) enfatizó la lucha contra la pobreza y la desigualdad. A pesar de mantenerse el carácter de excepcionalidad durante este periodo, se introdujeron cambios en la manera de gobernar el VRAEM. El cambio de estrategia implicó que en el periodo se comenzase a pensar a la población como 
objeto a gobernar. Así, se encarga al Instituto Nacional de Informática y Estadística (INEI) la generación de estadísticas específicas sobre la situación económica, educativa y sanitaria de la población del VRAEM (INEI, 2012). Cabe precisar que el VRAEM no es una división administrativa, por lo que el INEI no suele procesar información a esta escala. Así, este levantamiento de información se hizo en función de los distritos señalados en el Decreto Supremo 021-2008-DE-SG y, posteriormente, en el Decreto Supremo 040-2016-PCM. Ambos decretos delimitan tanto un territorio declarado en emergencia como una población sobre la cual trabajar.

Esta información se empleó para desplegar políticas sociales focalizadas en esta zona. Se multiplicó la presencia de programas sociales como Juntos ${ }^{8}$, Pensión 65 y Beca 18. Asimismo, se otorgó mayor independencia y recursos al órgano rector de la estrategia: la Comisión Multisectorial para la Pacificación y el Desarrollo Económico social en el VRAEM - CODEVRAEM. La CODEVRAEM mejoró su capacidad de liderazgo (aunque aún con limitaciones) y su proximidad con los habitantes locales. Es durante este periodo que se comenzaron a abrir nuevas oficinas de órganos de fiscalización tributaria, fiscalización ambiental, fiscalización energética, defensa al consumidor, así como oficinas del Ministerio de Inclusión Social (MIDIS), del Ministerio de la Mujer y Poblaciones Vulnerables (MIMP), Ministerio de Educación, apertura de institutos tecnológicos, sedes descentralizadas de la Universidad Nacional, entre otros. Además, se reforzó la presencia del Ministerio de Agricultura y de los programas productivos y de desarrollo alternativo (PROVRAEM, INIA, entre otros). Los ingenieros son el principal personaje de esta forma de gobernar a través de la difusión de saberes y tecnología, como ya lo habían hecho en el siglo XIX (Sala I Vila, 2006). Una vez más, son ellos los pioneros de la difusión del Estado civil en esta zona de la selva peruana.

${ }^{8}$ El Programa Juntos es el equivalente peruano al Programa "Bolsa Familia" de Brasil; se trata de programas de transferencias monetarias condicionadas. En el caso de este programa, se ha desarrollado un sistema de información específico que genera estadística para sus zonas de intervención priorizadas, entre ellas el VRAEM como se aprecia en http://www.juntos. gob.pe/modulos/mod_infojuntos/indexv.html 
A pesar de los temores de muchos funcionarios civiles por ir a una zona de emergencia, el Estado aumenta y diversifica su presencia en el quinquenio 2011-2016. Cada uno de los ministerios que conformaban la CODEVRAEM tuvo que elaborar un plan de intervención especifico (Mendoza; Leyva, 2017; MIDIS, 2013; MINAGRI, 2015) y mandar personal regularmente a la zona, incluso si no había abierto una oficina descentralizada en el valle.

Los cambios que sucedieron en este periodo permitieron reconocer una nueva forma de gobernar el VRAEM desde las instituciones Estatales, ya no solo controlando su territorio sino vinculándose con su población. Este cambio deja entrever que dos tipos de poder existen en la zona: el poder soberano y el poder gubernamental (Foucault, 2006). El Estado apuesta por aproximarse a la población a partir de prácticas de salud y alimentación, prácticas educativas, prácticas tributarias, prácticas de consumo, entre otras y generar conductas deseables a los ciudadanos de esta zona. Se reconoce así que los habitantes del VRAEM no son solo sujetos que controlar, sino también una población a la cual hay que ayudar a mejorar su vida. Varios de ellos se convierten en beneficiarios de programas sociales y servicios estatales. Esto significa un cambio sustancial en comparación con la estrategia anterior. Los programas tenían metas de cobertura y el discurso giraba en torno al desarrollo sostenible de la zona. La población también reconoce este cambio de estrategia, como lo menciona un antiguo alcalde del distrito Llochegua:

Desde mi punto de vista Humala hizo lo que no hizo [sic] otros presidentes desde los 70. El presidente empezó a comunicar, el empezó a soltar plata a las comunidades que estaba abandonados años y años; antes no teníamos infraestructura, carreteras, no teníamos electricidad, pero ahora ha mejorado la salud, lo que quisiera es que Humala repita unos 5 años más (César, Llochegua, enero de 2016).

Incluso las fuerzas armadas desarrollan cada vez más las denominadas "campañas sociales" que consisten en campañas itinerantes, en coordinación con otras instituciones estatales, de servicios médicos y, en algunos casos, 
también de justicia y otros para la población de diferentes distritos del VRAEM. Como se pudo observar durante el trabajo de campo, durante estas campañas los militares quedan en segundo plano, como apoyo logístico y de orientación a la población. Así, el equipamiento militar (helicópteros, carpas, raciones etc.) se usa para realizar estas campañas. El objetivo no es solo llevar salud a esta población sino indicarle como debe cuidar de sí misma en su vida cotidiana - se imparten consejos sobre alimentación para prevenir la anemia y la desnutrición, se realizan análisis de sangre para diagnosticar enfermedades crónicas, asimismo se les da consejos legales. En algunos casos también se les entrega donativos de vestimenta y alimentos.

El objetivo de las agencias gubernamentales era por lo tanto generar confianza en la población a fin de convencerlos de cambiar hacia una actividad lícita. La intención era acercarse más a la población, incluso en lo relacionado a la seguridad ciudadana, pero las capacidades institucionales todavía son un problema para la implementación. Como señala un policía en el distrito de Llochegua, cuando explicaba las dificultades para operar en la zona:

Esta es la única comisaria de este rincón de Huanta, de esta última parte del lado del VRAEM, hay zonas muy alejadas como el distrito de Mayapo que está como a media hora de aquí, otro, Canayre, que está a 50 minutos también le pertenece a esta jurisdicción, ahí también hay un cuartel, donde el ejército tiene un cuartel, pero ir ahí con una patrulla a hacer un servicio policial es prácticamente inaccesible, aparte de eso el riesgo seria alto porque ahí hay remanencia del terrorismo y también por la alta incidencia de narcotráfico en todo este valle (Jorge, Llochegua, enero de 2016).

El objetivo de esta gubernamentalización parece ser principalmente la población considerada por el Estado como "vulnerable", es decir, objeto de asistencia social, muchas veces en situación de pobreza o exclusión. En ese sentido, se desarrolla un Estado protector mínimo y no un Estado de bienestar generalizado para la población del VRAEM, lo que limita el alcance de este intento de gubernamentalización. 
El desarrollo de esta manera de gobernar parece apuntar a la convivencia entre dos tipos de prácticas estatales, por una parte un Estado soberano represor y por otra parte un Estado mínimamente protector y asistencialista. Esta convivencia de dos tipos de poderes parece no ser extraño en los países del denominado "Sur Global". Por el contrario, las realidades de estos países parecen justamente apuntar hacia esa dirección (ver por ejemplo Boege; Brown; Clements, 2009; Schlichte, 2005). En el caso peruano, la convivencia de dos tipos de poderes ya ha sido explorada para el caso del gobierno de Alan García en relación al surgimiento de una gubernamentalidad neoliberal (Drinot, 2011; 2014). Aplicar esta perspectiva teórica al VRAEM permite pensar el valle como un territorio en transición, e incluso en disputa, entre poder soberano y poder gubernamental.

\section{¿Un gobierno de sí para consigo en la emergencia?}

Como mencionamos anteriormente, el poder gubernamental se define tanto por su objeto, la población, como por la forma en la que se ejerce desde los individuos quienes se autorregulan. Ello implicaría que los objetivos de mejora son adoptados por la población para influenciar su propia conducta. No obstante, lo que pudimos observar durante el trabajo de campo es que esto no parece ser el caso con la totalidad de las prácticas de estatalidad en el VRAEM.

Existen algunas prácticas que vienen asentándose en la población local referidas a alimentación, vacunación así como prácticas productivas (técnicas de sembrío y mantenimiento de café y cacao). Los habitantes del VRAEM no se oponen a los mecanismos desplegados por el poder gubernamental, por el contrario, han adoptado su rol de beneficiarios y reclaman una mayor presencia de los componentes sociales del Estado. Incluso el representante de cocaleros en el VRAEM comparte esta alternativa de intervención para reducir la presencia de la coca:

Igual este pueblo necesita mayor inversión y mayor presencia del Estado. Imagínate, no hay banco de la nación, y no hay telefónica del Estado. imagínate 
acá no hay un hospital. Entonces creo que el Estado debe enfocarse más al VRAEM para que la población también vea a la presencia del Estado. De esta manera la producción de la hoja de coca baje de una o otra manera (Christian, Llochegua, enero de 2016).

Sin embargo, este proceso hacia un poder gubernamental no termina de tender puentes entre el Estado y la sociedad, como nos relató una funcionaria local en Pichari:

Hay incomprensión con el Estado. La gente aquí demanda siempre más presencia del Estado y más apoyo, pero cuando llegan las instituciones [...] y se hacen ferias informativas para informar a la ciudadanía sobre sus derechos a pocos les interesa. No muchos van. Muchos de los habitantes esperan que el Estado les regale cosas o les de ayuda. Es cierto que hay problemas de salud y educación, pero la gente no sabe apreciar que en Pichari hay instituto tecnológico, y universidad. Incluso la gente no siempre se anima a postular. Igual que con beca 18 que no siempre les interesa participar (Irene, Pichari, enero de 2016).

El modelo emprendedor y la superación por la educación también están presentes en los discursos y prácticas locales. La educación y la salud, así como la demanda por un Estado protector están en el centro de las preocupaciones. Así, por ejemplo, uno de los principales reclamos locales es la correcta implementación de la sede de la Universidad Nacional San Cristóbal de Huamanga (UNSCH), en Pichari, dedicada a la ingeniería agroforestal.

Incluso cuando el Estado no provee opciones para seguir estas prácticas, los habitantes del VRAEM han recurrido al sector privado para compensar las carencias de presencia estatal. Por ejemplo, tanto la oferta privada de educación (colegios, institutos) como la de salud (boticas, farmacias, consultorios médicos y odontológicos) se han expandido de manera exponencial en la última década. Además, existe una fuerte demanda de infraestructura en la zona. Así, cuando preguntamos por el principal rol de las autoridades locales, los ciudadanos locales respondieron que este rol es la promoción de inversiones por parte del Estado. 
Por otra parte, a pesar de que los habitantes del VRAEM viven en una constante ambigüedad frente a la Ley y el Estado en lo relacionado a temas de narcotráfico, no dejan de tener estos valores presentes. En ese sentido, varios de los entrevistados que señalaron haber tenido alguna participación en actividades ilegales manifiestan que reinvirtieron el dinero que ganaron en su educación para ser profesionales o en negocio legales:

A ver, yo soy cocalero. Gracias a la coca estoy tratando de educar a mis hijos para que mañana, más tarde, mis hijos no sean igual que yo. $Y$ yo quiero darles la mejor calidad de educación a ellos y mejor calidad de vida para que ellos más tarde sean útiles para la sociedad mejor que yo. (Christian, Llochegua, enero de 2016).

La apariencia de legalidad no es sólo un tema de fachada para evitar el control de las autoridades, se trata de una manera de definirse a sí mismos. Así, a pesar de que, en muchos casos, los habitantes del VRAEM dependen económicamente directa o indirectamente de una actividad ilegal, ninguno de los entrevistados se definió como narcotraficante. El involucramiento en el narcotráfico en el VRAEM es una cuestión de oportunidad y no una trayectoria criminal como también lo señalan otros estudios (Barrenechea; Tapia, 2016). La reinversión del dinero "sucio" en actividades productivas, la participación activa en la comunidad y la inserción en las dinámicas económicas legales de intercambio y consumo de manera pacífica resultan más importantes al momento de decidir cómo comportarse a nivel local.

En lo relacionado a temas de seguridad, hay una ambigüedad en cuanto al modelo jurídico político. En lo referente a temas de seguridad ciudadana, los comités de autodefensa organizados por los propios ciudadanos continúan patrullando los distritos del VRAEM para asegurar el orden, el imperio de la ley y lo que ellos consideran como moral. Los delitos patrimoniales y los delitos sexuales son condenados por la población y se piden sanciones fuertes e inmediatas, como lo explica una autoridad en Santa Rosa:

(H)ay todavía comunidades, organizaciones que están aplicando o sacan nuevas leyes internas para ellos nada más, por decir, hay otras personas que tienen 
problemas por violencia familiar u otros, entonces ellos mismos aplican esa ley ino?, tiran chicote, otras veces se envuelven con ortigas, son leyes internas, o le meten al calabozo, le hacen trabajar, castigan físicamente (David, Santa Rosa, enero de 2016).

Asimismo, algunos habitantes también colaboran con las fuerzas del orden para garantizar una mejor vigilancia del territorio, especialmente a través de las autodefensas. Los propios ciudadanos colaboran con el ejercicio del poder soberano o se substituyen a él cuando el Estado está ausente para hacer valer el orden legal en las materias que los preocupan. Las autodefensas se formaron en reacción al conflicto armado a inicios de los años 1980 s (1983). Inicialmente, actuaron como proveedores de seguridad y defensa contra Sendero Luminoso en un momento cuando todavía no había una presencia de Fuerzas Armadas en la región. Pronto, las autodefensas también proporcionaron seguridad dentro de las comunidades, incluso castigando quienes no seguían sus reglas. Así, fueron un actor clave en tiempos del conflicto armado interno. Hasta hoy, estos grupos de autodefensa son una fuente importante de orden y seguridad. Ello, conjuntamente con su rol histórico, forma las bases sobre las que se construye su legitimidad en la región. En muchas entrevistas, se dice que las autodefensas efectivamente promueven la seguridad, mientras que la policía no es capaz de hacerlo. Con ello las autodefensas son más legítimas en comparación con las fuerzas del orden:

Bueno, tenemos una comisaría; podemos decir que no está cumpliendo, a veces ellos vienen y están más dentro de su establecimiento, salen muy pocas veces en alguna reunión; la comunidad le ha dicho bien claro que acá no es muy útil la policía porque no cumple como debe ser. A veces sale, a veces no. Acá, quien cumple una función muy importante es el comité autodefensa de dar seguridad a toda la población; existe comité de autodefensa central y del cercado del pueblo de Llochegua, ellos si están las 24 horas al tanto, cuando hay problemas salen, cuando hay asaltos se van, manejan armamento permitido por el Estado, simple pero son más efectivos que los policías (Pedro, Llochegua, enero de 2016). 
Existe una marcada diferencia en la actitud de los actores e instituciones no estatales frente a diversos tipos de delitos, siendo más permisivos con algunas actividades que con otras. Esta ambigüedad es particularmente clara con respecto al narcotráfico, considerado como una "ilegalidad legitima" (Mayntz, 2016) o un ilegalismo. Así lo evidencia la actitud de los agricultores cocaleros quienes también son beneficiarios de los programas de reconversión de cultivos. Como se observó en el trabajo de campo, por una parte, los agricultores entregan sus parcelas de coca más antiguas y menos productivas para recibir los beneficios del programa, pero, por otro lado, continúan sembrando coca en otras parcelas más alejadas con la finalidad de venderla para la producción de drogas.

Ello es una muestra de la clase de comportamiento contradictorio de los habitantes del VRAEM, al menos contradictorio desde la perspectiva del Estado. Para ellos constituye una forma de maximizar sus posibilidades de ganancia y sobrevivencia. Este comportamiento deja dudas con relación al avance de la gubernamentalización del VRAEM y la sostenibilidad de la intervención estatal. Podemos afirmar que hay un intento de gubernamentalización, pero que no termina de consolidarse. Desde la perspectiva de Foucault, el poder es un elemento relacional, sin embargo, la relación de la gubernamentalización parece ser por el momento unidireccional desde el Estado central, de arriba hacia abajo.

\section{Conclusiones}

Desde la perspectiva del Estado, en el VRAEM existe una convivencia entre el poder gubernamental y el poder soberano, aunque no sin tensiones, especialmente en lo referente al narcotráfico. Como mencionaba Drinot (2011, p. 181) "es mejor pensar la dupla soberanía y gubernamentalidad como formas de poder conmensurables antes que como 'etapas' diferentes y sucesivas en la 'genealogía del Estado'". La preeminencia del enfoque del poder soberano combinado con el poder disciplinario ha dificultado que estas zonas sean completamente incluidas en lógicas de estatalidad. 
La convivencia entre los diferentes actores se complica, ya que no solo el Estado pretende ejercer el control de las conductas de los habitantes en el VRAEM. Los actores ilegales que operan en la zona se integran a las dinámicas locales, especialmente cuando no son violentos o cuando no son claramente identificables como criminales. Ello facilita la tolerancia a este tipo de actores en contraste con el rechazo de ciertos actores gubernamentales. Además, permite que los actores criminales apliquen ciertas reglas que son respetadas a nivel local, aunque no necesariamente haya una organización criminal detrás que centralice la aplicación de estas reglas.

Así, se puede pensar que en este tipo de zonas existen ordenes híbridos (Boege et al., 2009) en donde los normas y actores del Estado coexisten con aquellos actores y normas no-estatales e ilegales. Correspondería asimismo explorar la posibilidad de que los actores ilegales también ejerzan poder gubernamental e incluso soberano, como lo han sugerido algunos autores (Nieto, 2012; Sheptycki, 2003).

Los habitantes del VRAEM eligen las reglas por las cuales son gobernados en ciertas esferas de su vida. Así, en función de sus propios intereses, deciden adherir u oponerse a lo planteado por el Estado en la zona, pero también aceptan su presencia y reglas en otras ocasiones. Por lo tanto el Estado está presente en el VRAEM y su crecimiento revela un fortalecimiento de sus capacidades. Los actores estatales que ejercen los dos tipos de poder, conscientes de esta situación, conviven pero también compiten por recursos, autoridad y respeto de la población. Esto ha llevado a algunas pugnas sobre quien coordina los esfuerzos que despliega el Estado en la zona.

Esto probablemente refleje el carácter coyuntural de las características del Estado en el VRAEM. A pesar de existir planes y estrategias planteadas periódicamente, los resultados del ejercicio efectivo del poder gubernamental y del poder soberano distan de corresponder a las expectativas estatales y terminan adoptando sus propias características en base a la reacción de los habitantes a nivel local. ¿Cómo se gobierna el VRAEM? Como sus habitantes aceptan, en algunos temas o son efectivamente forzados, en otros, a ser gobernados. Dado el déficit de capacidades estatales para cubrir una 
presencia efectiva en todo el territorio, el carácter voluntario de la aplicación de ciertas prácticas de gobierno es de vital importancia para que el Estado siga presente incluso allí dónde está materialmente ausente. La relación de la población al Estado se guía también por la esperanza de que una mayor inclusión resulte en mayor desarrollo económico. Esta esperanza se diluye cuando el Estado aplica un enfoque orientado a la destrucción del principal motor de la economía: el narcotráfico. Por otra parte, la visión asistencialista del Estado evidencia que en el gobierno domina una visión de arriba hacia abajo, sin necesariamente incluir a la sociedad en el proceso.

Estos aspectos podrían ser explorados en detalle por futuros estudios comparados sobre regiones con un bajo nivel de control estatal y una fuerte presencia de economías ilícitas. Es un esfuerzo que se viene realizando en estudios que abordan la relación entre una economía ilícita y el Estado en zonas similares como en Colombia, México o Afganistán, siendo necesario multiplicar los casos y las experiencias como las del VRAEM a este tipo de comparaciones. La exploración evidencia que un punto clave que limita el desarrollo de una gubernamentalidad en el VRAEM es el excesivo enfoque del Estado en el problema de las drogas, lo que limita el desarrollo de una relación entre el Estado y la sociedad. En esta perspectiva, la sociedad sigue siendo un objeto a gobernar en lugar de estar incluida en un proceso de desarrollo integral.

Sofia Isabel Vizcarra Castillo es Doctora en Sociología y docente del Departamento Académico de Ciencias Sociales de la Pontificia Universidad Católica del Perú. Actualmente es investigadora de pós-doctorado en el Departamento de Economía y Relaciones Internacionales de la Universidad Federal de Rio Grande do Sul.

$\equiv$ sofia.vizcarra@pucp.edu.pe

Christoph Heuser es investigador en el Instituto GIGA de Estudios Latinoamericanos. Su investigación se centra en las economías ilícitas, el crimen organizado y la formación del estado, así como en la construcción del estado en regiones marginales.

”christoph.heuser@fes.de 


\section{Referencias}

1. ADORNO, Sérgio. Perturbações: Foucault e as ciências sociais. Sociologia e Antropologia, v. 7, n. 1, p. 33-61, 2017. doi: 10.1590/2238-38752017v712

2. AGAMBEN, Giorgio. State of exception. Chicago: The University of Chicago Press, 2005.

3. ARIAS, Enrique D. Criminal enterprises and governance in Latin America and the Caribbean. Cambridge: Cambridge University Press, 2017. doi: $10.1017 / 9781316650073$

4. ARJONA, Ana. Rebelocracy: social order in the Colombian Civil War. Cambridge: Cambridge University Press, 2017. doi: 10.1017/9781316421925

5. BARRENECHEA, Laura; TAPIA, Luis. Mochileros: una carga pesada. Lima: CEDRO - Centro de Información y Educación para la Prevención del Abuso de Drogas, 2016. http://repositorio.cedro.org.pe/bitstream/CEDRO/309/1/ Mochileros.pdf

6. BOEGE, Volker; BROWN, Anne M.; CLEMENTS, Kevin P. Hybrid political orders, not fragile states. Peace Review, v. 21, n. 1, p. 13-21, 2009. doi: 10.1080/10402650802689997

7. CALMET, Yasmin; SALAZAR, Diego. VRAEM: políticas de seguridad pública en zona de conflicto. Cuadernos de Marte, v. 4, n. 5, p. 157-186, 2013. https:// dialnet.unirioja.es/servlet/articulo?codigo $=6114269$

8. CARPIO, David F. S. V. del; ANNONI, Danielle. El valle de los ríos Apurímac, Ene y Mantaro - VRAEM: entre el narcoterrorismo y el descaso del estado peruano. Nuevo Derecho, v. 12, n. 18, p. 107-120, 2016.

9. CEPLAN - Centro Nacional de Planeamiento Estratégico. Plan Integral Territorial VRAEM: 2012-2016. Lima: CEPLAN, 2012.

10. DARGENT, Eduardo. El estado en el Perú: una agenda de investigación. Lima: Pontificia Universidad Católica del Perú, 2012.

11. DAS, Veena; POOLE, Deborah. Anthropology in the margins of the state. Nova York: Oxford University Press, 2004.

12. DONADIO, Marcela (Org.) Perú. In: Atlas Comparativo de la Defensa en América Latina y Caribe - Edición 2016. Buenos Aires: Red de Seguridad y Defensa de América Latina - RESDAL, 2016. p. 194-197.

13. DRINOT, Paulo. The meaning of Alan García: sovereignty and governmentality in neoliberal Peru. Journal of Latin American Cultural Studies: Travesia, v. 20, n. 2, p. 179-195, 2011. doi: 10.1080/13569325.2011.588514

14. DRINOT, Paulo (Ed.). Peru in theory. New York: Palgrave Macmillan, 2014.

15. DUNCAN, Gustavo. Una lectura política de Pablo Escobar. Co-herencia, v. 10, n. 19, p. 235-262, 2013. doi: 10.17230/co-herencia.10.19.9 
16. FELBAB-BROWN, Vanda; TRINKUNAS, Harold; HAMID, Shadi. Militants, criminals, and warlords. Washington D.C: Brookings Institution Press, 2017. https://www.jstor.org/stable/10.7864/j.ctt1w6t9cd

17. FOUCAULT, Michel. La ética del cuidado de sí como práctica de la libertad. Nombres, Revista de Filosofía, v. X, n. 15, p. 257-280, 2000.

18. FOUCAULT, Michel. Seguridad, territorio, población. Buenos Aires: Fondo de Cultura Económica, 2006.

19. GARCÍA ROMANUTTI, Hernán. El Estado según Foucault: soberanía, biopolítica y gubernamentalidad. Utopía y Praxis Latinoamericana, v. 19, n. 66, p. 53-66, 2014. http://www.redalyc.org/articulo.oa?id=279/27937089006

20. GARMANY, Jeff. The embodied state: governmentality in a Brazilian favela. Social \& Cultural Geography, v. 10, n. 7, p. 721-739, 2009. doi: 10.1080/14649360903205132

21. HANAFI, Sari; LONG, Taylor. Governance, governmentalities, and the state of exception in the Palestinian refugee camps of Lebanon. Journal of Refugee Studies, v. 23, n. 2, p. 134-159, 2010. doi: 10.1093/jrs/feq014

22. INEI - Instituto Nacional de Estadística e Informática. VRAEM- Ámbito de Influencia. Ámbito de Intervención. Lima: INEI, 2012.

23. IPSOS. Revocatoria, indulto y terrorismo. Opinión data. Resumen de Encuestas a la opinión pública. v. 12, n. 159, 19 nov. 2012.

24. LESSING, Benjamin. Making peace in drug wars: crackdowns and cartels in Latin America. Cambridge: Cambridge University Press, 2017. doi: 10.1017/9781108185837

25. MAYNTZ, Renate. Illegal markets: boundaries and interfaces between legality and illegality. MPIfG Discussion Paper 16/4, Max Planck Institute for the Study of Societies, 2016. https://ideas.repec.org/p/zbw/mpifgd/164.html

26. MENDOZA, Waldo; LEYVA, Janneth. La economía del VRAEM. Lima: USAID/ CIES, 2017.

27. MIDIS - Ministerio de Desarrollo e Inclusión Social. Informe final de la Comisión Quipu para el VRAEM. Lima: MIDIS, 2013.

28. MINAGRI - Ministerio de Agricultura y Riego. Principales indicadores del Valle de los ríos Apurímac, Ene y Mantaro - VRAEM. Lima: INEI, 2015. http:// minagri.gob.pe/portal/download/pdf/interes/agrovraem/anexo2_prin-indicadores. pdf

29. MONTEAGUDO, Manuel-Antonio. EI VRAEM que no vemos. Revista Ideele, v. 232, p. 1-5, 2013.

30. NIETO, Diego. Neoliberalismo, biopolítica y gobernanza del crimen transnacional. Colombia Internacional, n. 76, p. 137-165, 2012. doi: 10.7440/ colombiaint76.2012.06

31. O'DONNELL, Guillermo. 1993. "On the State, Democratization and Some Conceptual Problems: A Latin American View with Glances at Some Postcommunist 
Countries." World Development, SPECIAL ISSUE, 21 (8):1355-69. https://doi. org/10.1016/0305-750X(93)90048-E.

32. PANSTERS, Wil G. Drug trafficking, the informal order, and caciques. Reflections on the crime-governance nexus in Mexico. Global Crime, v.19, p. 1-24, 2018. doi: 10.1080/17440572.2018.1471993

33. PAREDES CASTRO, Juan. El regalo del VRAEM, la columna de Juan Paredes Castro. El Comercio, Lima, 10 set. 2017. https://elcomercio.pe/politica/regalovraem-columna-juan-paredes-castro-noticia-456964

34. SALA I VILA, Núria. Ingenieros y colonización amazónica en el Perú, 1821 1930. Anuario IEHS, v. 21, p. 441-466, 2006.

35. SCHLITCHE, Klaus. Der Staat in der Weltgesellschaft: Politische Herrschaft in Asien, Afrika und Lateinamerika. Frankfurt: Campus, 2005.

36. SCHMITT, Carl. Politische Theologie: Vier Kapitel zur Lehre von der Souveränität. Berlin: Duncker \& Humblot, 1996.

37. SHEPTYCKI, James. The governance of organised crime in Canada. Canadian Journal of Sociology / Cahiers Canadiens de Sociologie, v. 28, n. 4, p. 489 - 516, 2003. doi: 10.2307/3341839

38. SILES VALLEJOS, Abraham. La emergencia... en el corazón del constitucionalismo peruano: paradojas, aporías y normalización. Thémis - Revista de Derecho, v. 67, p. 73-84, 2015.

39. UNODC - United Nations Office on Drugs and Crime. Monitoreo de cultivos de coca 2016. Lima: UNODOC/ DEVIDA, 2017.

40. VIZCARRA, Sofía. El impacto de los problemas de implementación del régimen de control de insumos químicos en la cadena del tráfico ilícito de drogas entre los años 2007 y 2011 en las principales cuencas cocaleras del Perú. Tesis [Maestría en Ciencia Política] 2014. 92 f. Pontificia Universidad Católica del Perú, 2014.

Recibido en: 09 nov. 2018

Aceptado en: 07 mayo. 2019 
